\title{
The function of androgen/androgen receptor and insulin growth factor-1/insulin growth factor-1 receptor on the effects of Tribulus terrestris extracts in rats undergoing high intensity exercise
}

\author{
YIN WU $^{1}$, HONGFANG YANG $^{2}$ and XIAOHUI WANG ${ }^{2}$ \\ ${ }^{1}$ Department of Computer and Statistics and ${ }^{2}$ School of Kinesiology, Shanghai \\ University of Sport, Shanghai 200438, P.R. China
}

Received January 25, 2016; Accepted May 9, 2017

DOI: $10.3892 / \mathrm{mmr} .2017 .6891$

\begin{abstract}
Our previous study demonstrated that treatment with Tribulus terrestris (TT) extracts $(120 \mathrm{mg} / \mathrm{kg})$ promoted the muscle weight gain and performance of rats undergoing high intensity exercise. The present study was designed to explore the mechanisms underlying the effect of treatment with TT extracts and the involvement of androgens, the androgen receptor (AR), insulin growth factor-1 (IGF-1) and the IGF-1 receptor (IGF-1R). A total of 32 Sprague-Dawley rats were randomly divided into groups as follows: Control; TT, treated with TT extracts, E, high intensity exercise; $\mathrm{E}+\mathrm{TT}$, high intensity exercise plus TT treatment. The rats of the $\mathrm{E}$ and $\mathrm{E}+\mathrm{TT}$ groups underwent high intensity exercise with a progressively increasing load for 5 weeks, and TT extracts were intragastrically administered in the TT and $\mathrm{E}+\mathrm{TT}$ rats $30 \mathrm{~min}$ prior to training. TT extract composition was analyzed using ultra-high performance liquid chromatography-quadrupole-time of flight mass spectrometry. Testosterone and IGF-1 plasma levels and AR, IGF-1R and myosin heavy chain (MHC) protein levels in muscles were determined by ELISA and western blotting, respectively. The saponins tigogenin and diosgenin comprised $\sim 71.35 \%$ of the total peak area. Compared with the E group, TT extracts increased the testosterone and IGF-1 plasma levels, and $\mathrm{AR}, \mathrm{IGF}-1 \mathrm{R}$ and MHC protein levels in the gastrocnemius of rats undergoing high intensity exercise, accompanied
\end{abstract}

Correspondence to: Professor Xiaohui Wang, School of Kinesiology, Shanghai University of Sport, 650 Qingyuanhuan Road, Shanghai 200438, P.R. China

E-mail:wangpan96@126.com

Abbreviations: TT, Tribulus terrestris; AR, androgen receptor; IGF-1, insulin growth factor-1; IGF-1R, IGF-1 receptor; MHC, myosin heavy chain; UHPLC-Q-TOF/MS, ultra-high performance liquid chromatography-quadrupole-time of flight mass spectrometry

Key words: testosterone, AR, IGF-1R, IGF-1, Tribulus terrestris extracts, high intensity exercise with increased body weight and gastrocnemius weight. In conclusion, the effect of TT extracts on the performance of high intensity exercise rats may be attributed to increased levels of circulating testosterone and IGF-1 and increased AR and IGF-1R protein expression levels in the gastrocnemius, resulting in increased muscle weight and increased $\mathrm{MHC}$ in the gastrocnemius. The present study provided preliminary evidence supporting the use of TT extracts as a dietary supplement for the promotion of skeletal muscle mass increase and the enhancement of athletic performance in humans performing high intensity exercise.

\section{Introduction}

Androgens are anabolic hormones that, in addition to their androgenic activity, are involved in the regulation of metabolic function, energy utilization, carbohydrate metabolism, fat metabolism and nitrogen retention in several extragenital tissues (1). High endogenous concentrations of testosterone induce increases in muscle size and strength (2) and confer physiological and psychological advantages in sports (3). Therefore, androgenic-anabolic steroids (AAS) are widely abused by athletes (4). However, the side effects to organs and systems that result from androgenic-anabolic steroid abuse should be addressed. These systems include the cardiovascular system, the central nervous system and the male and female reproductive systems (5). Certain androgenic herbs may be able to mimic the endocrine signaling process in the body and increase serum androgen levels, such as Tribulus terrestris (TT) (6-9).

As a well-known traditional Chinese medicine, TT extracts have been used in China and India for the treatment of multiple disorders due to its range of properties, which include aphrodisiac effects $(9,10)$, protective effects against ischemia/reperfusion injury, anticancer and antihypertensive properties (10). TT extracts, which are primary saponins, may be used by athletes and bodybuilders who are concerned about increasing muscle mass, strength and improving performance, even if the underlying biological pathways remain to be determined. The primary effect of TT is claimed to be an increase in anabolic and androgenic function through the activation 
of endogenous testosterone production, providing a natural, safe and legal method to increase testosterone levels with no side effects $(6,9,11,12)$. However, several research groups have previously reported that TT extracts have no significant effect on the performance and plasma levels of testosterone in intact and castrated male rats (13), in rugby athletes (14), men undergoing resistance training $(15,16)$ and untrained females $(17)$. However, a previous study demonstrated that TT extracts promoted the performance of rats undergoing high intensity exercise for 5 weeks, by preventing a heavy training-induced decrease in serum testosterone (18). The aim of the present study was to further determine the underlying mechanisms.

Testosterone exerts its biological functions primarily via the androgen receptor (AR), which is a member of the ligand-dependent transcription factor superfamily (19). Upon binding to androgens, cytosolic AR translocates into the nucleus and binds to the promotor/enhancer regions of specific DNA sequences known as the androgen response elements. It then regulates target gene transcription by recruiting coactivators or corepressors. Exercise is involved in regulating testosterone secretion along with AR expression and activity. In general, testosterone levels are increased following exercise, in particular resistance training in men; however, testosterone levels are reduced in response to high intensity training and overtraining $(3,20,21)$. As for AR, exercise increases AR binding capacity in a skeletal muscle fiber type-dependent manner. Endurance training leads to a significant increase in AR binding capacity in the soleus (composed of predominantly slow twitch muscle fibers), whereas resistance training elicits a significant increase in the extensor digitorumlongus (composed of predominantly fast twitch muscle fibers). Exercise also increases AR protein expression levels. Lee et al (22) demonstrated that AR protein concentration increased by 106 and $279 \%$ in rat plantaris muscle following 7 and 21 days of overload exercise, respectively, and AR mRNA expression levels increased by $430 \%$ following 7 days of exercise. Exercise-induced upregulation of AR content was also observed in female rats (23) and aging men (24). However, it has also been reported that AR protein levels in the vastus lateralis decreased $(20,25)$ or remained unchanged (26) following resistance exercise.

Insulin-like growth factor-1 (IGF-1), a peptide hormone, is involved in promoting muscle hypertrophy, muscle repair and alleviating muscle damage. The primary effect of IGF-1 is mediated by binding to the IGF-1 receptor (IGF-1R), a widely-expressed cell surface heterotetramer that is similar to the insulin receptor. IGF-1 reduced age-associated wasting of skeletal muscle (27). Resistance training, which is the most useful treatment for the loss of muscle mass and strength in aging people, was determined to upregulate IGF-1 and IGF-1R expression levels (28). IGF-1R is involved in mediating physiological cardiomyocyte hypertrophy, as evidenced by the prevention of the hypertrophic response to swim training in cardiomyocyte-specific IGF-1R knockout mice (29). Androgen response elements are located within the promoter regions of IGF-1 and IGF-1R and testosterone administration in humans and rodents increases circulating and skeletal muscle IGF-1 levels. Testosterone deficiency is also associated with reduced levels of IGF-1 in humans. Therefore, IGF-1 and IGF-1R are the target genes of AR. In addition, the enhancing effect of testosterone on the proliferation of primary human skeletal muscle cells in vitro was blocked by small interfering RNA targeting human IGF-1R (30), indicating the existence of cross-talk between androgens and IGF-1R. Androgens are known to cross-talk with multiple signal transduction pathways, including the growth hormone/IGF-1 axis (31), the phosphatidylinositol 3-kinase/protein kinase B pathway (32), the Wnt/b-catenin signaling pathway (33) and Notch signaling (34). However, the mechanisms by which circulating IGF-1 and muscular IGF-1R mediate the effects of testosterone on the muscle remain to be fully understood. The aim of the present study was to detect the expression of AR and IGF-1/IGF-1R following TT extract-induced testosterone increases in rats undergoing high intensity exercise, and its relationship with the promoted performance.

\section{Materials and methods}

Animals, exercise protocol and administration of TT extracts. A total of 32 male Sprague-Dawley rats (8 weeks old; 210-220 g body weight), obtained from the Animal Center of The Second Military Medical University (Shanghai, China) were maintained at a controlled temperature $\left(22-24^{\circ} \mathrm{C}\right)$ and humidity (45-55\%) under a 12-h light/dark cycle, with free access to food and water. The rats were randomly divided into 4 groups, each with 8 rats: i) Control group; ii) TT, TT group; iii) E, high intensity exercise group; and iv) E+TT, high intensity exercise group plus TT. The E and E+TT group rats underwent high intensity training for a period of 5 weeks, as previously described (18). The TT and E+TT group rats were given TT extracts intragastrically $(120 \mathrm{mg} / \mathrm{kg})$ as in a previous study (18) $30 \mathrm{~min}$ prior to exercise training. Equal amounts of normal saline were administered to the non-TT treated groups. The animal protocol was approved and the experiments were supervised by the Ethics Committee of Shanghai University of Sport (approval no: 2012008).

Composition of TT extracts. TT extracts (saponins $>70 \%$ ) were purchased from Shanxi Huike Botanical Development Company (Shaanxi, China). The TT extract powder was dissolved in $70 \%$ ethanol and the supernatant was obtained by ultrasonic extraction and centrifugation at 20,217 $\mathrm{x} g$ for $10 \mathrm{~min}$ at $4^{\circ} \mathrm{C}$. The composition was determined using ultra-high performance liquid chromatography-quadrupole-time of flight mass spectrometry (UHPLC-Q-TOF/MS) revised according to the protocol of Wu et al (35) by Dr Xin Dong (College of Pharmacy, The Second Military Medical University, Shanghai, China).

Hormone assays. In order to avoid the acute effect of exercise on testosterone and IGF-1 plasma levels, rats were sacrificed $36 \mathrm{~h}$ following the last exercise session. Blood samples were collected and testosterone (cat no. KGE010) and IGF-1 (cat no. MG100) plasma levels were determined by ELISA using commercially available kits from R\&D Systems, Inc. (Minneapolis, MN, USA), according to the manufacturer's protocol.

Relative weight and protein content of gastrocnemius and soleus. The rat gastrocnemius and soleus muscles $(50 \mathrm{mg})$ 
were cut into sections and homogenized on ice with $500 \mu \mathrm{l}$ radioimmunoprecipitation assay lysis buffer and $1 \%$ phenylmethylsulfonyl fluoride (ShenergyBiocolor Bioscience and Technology Co., Ltd., China) in a homogenizer to extract total protein. The lysates were briefly sonicated on ice, and centrifuged at $11,963 \times \mathrm{g}$ for $10 \mathrm{~min}$ at $4^{\circ} \mathrm{C}$. Supernatants were collected and protein concentration was measured using a BCA protein assay kit (Pierce; Thermo Fisher Scientific, Inc., Waltham, MA, USA), according to the manufacturer's protocol.

Western blot analysis. Gastrocnemius and soleus extracts $(100 \mu \mathrm{g})$ were resolved by $10 \%$ SDS-PAGE and transferred to a nitrocellulose membrane. The membrane was blocked with $5 \%$ non-fat milk at $4^{\circ} \mathrm{C}$ overnight. The nitrocellulose membranes were probed overnight at $4^{\circ} \mathrm{C}$ with the following primary antibodies: Anti-AR (cat no. sc-816; 1:200), anti-IGF-1R (cat no. sc-7952; 1:500), anti-myosin heavy chain (MHC; cat no. sc-58797; 1:500; Santa Cruz Biotechnology, Inc., Dallas, TX, USA) and anti-GAPDH (cat no. SAB-2100894; 1:2,000; Sigma-Aldrich; Merck KGaA, Darmstadt, Germany). The blots were washed in TBS containing $0.1 \%$ Tween-20 and incubated at room temperature for $2 \mathrm{~h}$ with the following horseradish peroxidase-conjugated secondary antibodies at a 1:5,000 dilution: Goat anti-rabbit immunoglobulin (Ig) G (cat no. sc-2004), donkey anti-goat (cat no. sc-2020) and goat anti-mouse (cat no. sc-2005), obtained from Santa Cruz Biotechnology, Inc. Following washing with TBST, the blots were developed using enhanced chemiluminescence (cat no. WBKLS0100; Merck KGaA) and exposed to X-ray film to visualize the protein bands. The density of bands was determined using Tanon Bio-Image software version 1.00 (Tanon Science and Technology Co., Ltd., Shanghai, China). Experiments were performed in triplicate.

Statistical analysis. All values were expressed as the mean \pm standard deviation of 3 independent experiments. $\mathrm{P}<0.05$ was considered to indicate a statistically significant difference. Data were analyzed using one-way analysis of variance and Fisher's Least Significant Difference post-hoc test with SPSS software version 20.0 (IBM Corp., Armonk, NY, USA).

\section{Results}

Composition of $T T$ extracts, determined by UHPLC-Q-TOF/MS. The composition of the TT extracts was detected using UHPLC-Q-TOF/MS. The most abundant constituents were tigogenin and diosgenin, which are saponins and accounted for $\sim 60.19$ and $11.16 \%$ of the total peak area, respectively (Table I). No AAS were present in the TT extracts.

TT extracts attenuated high intensity exercise-induced weight gain in rats. There was no significant difference in weight gain between the control and TT groups (Table II). A significant reduction in body weight in the $\mathrm{E}$ and $\mathrm{E}+\mathrm{TT}$ groups was observed from the third week of training onwards, compared with the control group (week $3, \mathrm{P}<0.05$ and $\mathrm{P}<0.05$, respectively; week 4, $\mathrm{P}<0.01$ and $\mathrm{P}<0.01$, respectively, week $5, \mathrm{P}<0.01$ and $\mathrm{P}<0.01$, respectively; Table II). Treatment with TT extracts significantly attenuated the effect of high intensity training on weight gain compared with the E group at week $5(\mathrm{P}<0.05$; Table II).

Effects of TT extract treatment on the relative weight, protein concentration and protein levels of MHC in the gastrocnemius and soleus of rats undergoing high intensity exercise. Exercise-induced increases of the relative weight of the gastrocnemius and soleus and significantly increased relative weight of the gastrocnemius in the E+TT group compared with the control $(\mathrm{P}<0.05$; Fig. 1A). The relative weights of gastrocnemius and soleus did not significantly differ between the TT group and the control group or in the E+TT group compared with the E group (Fig. 1A and B), suggesting that TT extracts do not affect the relative weight of muscle in sedentary or exercising rats. The protein content of the gastrocnemius in the $\mathrm{E}$ and $\mathrm{E}+\mathrm{TT}$ group rats was significantly increased compared with the control group $(\mathrm{P}<0.05$ and $\mathrm{P}<0.01$, respectively; Fig. 1C), and there was a significantly greater increase in the $\mathrm{E}+\mathrm{TT}$ group compared with the $\mathrm{E}$ group $(\mathrm{P}<0.05$; Fig. $1 \mathrm{C})$. Soleus protein content did not significantly differ between the groups (Fig. 1D). Similar results were observed for MHC, with visible increases of gastrocnemius MHC protein expression levels observed in the TT and E+TT groups compared with the control group (Fig. 1E). MHC levels were significantly increased in the E+TT group compared with the control group and the $\mathrm{E}$ group $(\mathrm{P}<0.01$ and $\mathrm{P}<0.01$, respectively; Fig. $1 \mathrm{~F})$. No difference in soleus MHC protein expression level was detected between the groups (Fig. 1E and F).

No difference was observed in the relative weight, protein concentration or the protein levels of MHC in the soleus following treatment with TT extracts, and TT extract-induced increases in MHC protein concentration and expression levels were only observed in the gastrocnemius of the E+TT group rats compared with the E group. As a result, the gastrocnemius was used for the following studies as opposed to the soleus.

Effects of TT extracts on plasma testosterone levels and gastrocnemius AR protein levels in rats undergoing high intensity exercise. Following 5 weeks of high intensity exercise, plasma testosterone levels in E group were significantly decreased to $\sim 40 \%$ of the control $(\mathrm{P}<0.01$; Fig. $2 \mathrm{~A})$. This was attenuated by treatment with TT extracts and significantly increased plasma testosterone levels were observed in the $\mathrm{E}+\mathrm{TT}$ group compared with the E group $(\mathrm{P}<0.01$; Fig. 2A) and no significant difference between the E+TT group and the control (Fig. 2A). Gastrocnemius AR protein levels were significantly increased in the $\mathrm{E}$ and $\mathrm{E}+\mathrm{TT}$ groups compared with the control $(\mathrm{P}<0.05$ and $\mathrm{P}<0.01$, respectively; Fig. $2 \mathrm{~B})$ and the increase was greater in the $\mathrm{E}+\mathrm{TT}$ group compared with the E group ( $\mathrm{P}<0.05$; Fig. 2B).

Effects of TT extracts on plasma IGF-1 levels and gastrocnemius IGF-1R protein levels in rats undergoing high intensity exercise. Following 5 weeks of high intensity exercise, the plasma levels of IGF-1 in the E group rats remained unaltered compared with the control group rats (Fig. 3A). TT extracts increased the plasma levels of IGF-1 in the E+TT group compared with the control group and the E group $(\mathrm{P}<0.05$ and 
Table I. Ultra-high performance liquid chromatography-quadrupole-time of flight mass spectrometry analysis results of Tribulus terrestris extract components.

\begin{tabular}{rcccccccr}
\hline No. & Name & $\begin{array}{c}\mathrm{RT} \\
(\mathrm{min})\end{array}$ & Formula & $\mathrm{M} \pm \mathrm{X}$ & $\begin{array}{c}\text { Expected } \\
\mathrm{m} / \mathrm{z}\end{array}$ & $\begin{array}{c}\text { Experimental } \\
\mathrm{m} / \mathrm{z}\end{array}$ & $\begin{array}{c}\text { Error } \\
(\mathrm{ppm})\end{array}$ & $\begin{array}{r}\text { Peak } \\
\text { area }(\%)\end{array}$ \\
\hline 1 & Valine & 0.730 & $\mathrm{C}_{5} \mathrm{H}_{11} \mathrm{NO}_{2}$ & $\mathrm{M}+\mathrm{H}$ & 118.0862 & 118.0863 & 0.41 & 0.32 \\
2 & $\mathrm{~A}$ & 10.851 & $\mathrm{C}_{9} \mathrm{H}_{19} \mathrm{NO}$ & $\mathrm{M}+\mathrm{H}$ & 158.1541 & 158.1539 & -1.21 & 5.56 \\
3 & $\mathrm{~B}$ & 11.079 & $\mathrm{C}_{14} \mathrm{H}_{24} \mathrm{~N}_{4}$ & $\mathrm{M}+\mathrm{H} / \mathrm{M}+\mathrm{Na}$ & $249.2079 / 271.1902$ & $249.2074 / 271.1893$ & $-1.98 /-3.46$ & 5.45 \\
4 & Diosgenin & 12.620 & $\mathrm{C}_{27} \mathrm{H}_{42} \mathrm{O}_{3}$ & $\mathrm{M}+\mathrm{H}$ & 415.3215 & 415.3207 & -3.15 & 11.16 \\
5 & $\mathrm{C}$ & 12.685 & $\mathrm{C}_{31} \mathrm{H}_{35} \mathrm{~N}_{2} \mathrm{O}_{6}$ & $\mathrm{M}+\mathrm{H} / \mathrm{M}+\mathrm{Na}$ & $532.2577 / 554.2393$ & $532.2568 / 554.2387$ & $-0.98 /-1.51$ & 3.43 \\
6 & $\mathrm{D}$ & 12.905 & $\mathrm{C}_{23} \mathrm{H}_{30} \mathrm{O}_{6}$ & $\mathrm{M}+\mathrm{H} / \mathrm{M}+\mathrm{Na}$ & $403.2126 / 425.1938$ & $403.2115 / 425.1935$ & $-0.92 /-2.76$ & 9.11 \\
7 & Tigogenin & 13.492 & $\mathrm{C}_{27} \mathrm{H}_{44} \mathrm{O}_{3}$ & $\mathrm{M}+\mathrm{H}$ & 417.3369 & 417.3363 & -1.34 & 60.19 \\
8 & $\mathrm{E}$ & 13.842 & $\mathrm{C}_{30} \mathrm{H}_{32} \mathrm{O}_{8}$ & $\mathrm{M}+\mathrm{H} / \mathrm{M}+\mathrm{Na}$ & $521.2150 / 543.2009$ & $521.2170 / 543.1989$ & $-3.81 /-3.63$ & 4.73 \\
9 & Rutin & 7.660 & $\mathrm{C}_{27} \mathrm{H}_{30} \mathrm{O}_{16}$ & $\mathrm{M}+\mathrm{H}$ & 611.1608 & 611.1607 & -0.21 & 0.02 \\
10 & Kaempferol- & 8.332 & $\mathrm{C}_{21} \mathrm{H}_{20} \mathrm{O}_{11}$ & $\mathrm{M}+\mathrm{H}$ & 449.1071 & 449.1078 & 1.57 & 0.02 \\
& 3-glucoside & & & & & & & \\
11 & Quercetin & 9.380 & $\mathrm{C}_{15} \mathrm{H}_{10} \mathrm{O}_{7}$ & $\mathrm{M}+\mathrm{H} / \mathrm{M}-\mathrm{H}$ & 303.0507 & 303.0499 & -2.53 & 0.01 \\
\hline
\end{tabular}

RT, retention time; m/z, mass-to-charge ratio; A-E, uncertain materials from the database of TT extracts.

Table II. TT extracts enhanced high intensity exercise-induced weight gain in rats.

\begin{tabular}{lcccc}
\hline Week & Control & E & TT & E+TT \\
\hline 0 & $240.18 \pm 3.69$ & $236.23 \pm 9.58$ & $242.8 \pm 14.04$ & $228.73 \pm 2.05$ \\
1 & $273.8 \pm 7.02$ & $263.51 \pm 11.31$ & $276.62 \pm 16.27$ & $252.73 \pm 4.38$ \\
2 & $308.08 \pm 11.61$ & $295.8 \pm 23.02$ & $307.34 \pm 15.4$ & $294.93 \pm 5.36$ \\
3 & $351.48 \pm 18.25$ & $324.66 \pm 25.27^{\mathrm{a}}$ & $345.34 \pm 15.28$ & $324.23 \pm 7.61^{\mathrm{a}}$ \\
4 & $359.25 \pm 38.04$ & $322.4 \pm 25.27^{\mathrm{b}}$ & $380.12 \pm 16.45$ & $317.23 \pm 15.68^{\mathrm{b}}$ \\
5 & $402.35 \pm 30.45$ & $310.7 \pm 21.23^{\mathrm{b}}$ & $402.06 \pm 21.23$ & $327.15 \pm 15.75^{\mathrm{b}, \mathrm{c}}$ \\
\hline
\end{tabular}

E, high intensity exercise; TT, Tribulus terrestris extracts; E+TT, high intensity exercise plus TT. ${ }^{a} \mathrm{P}<0.05$ and ${ }^{\text {b }} \mathrm{P}<0.01$ vs. control. ${ }^{\mathrm{c}} \mathrm{P}<0.05$ vs . E.

$\mathrm{P}<0.05$, respectively; Fig. 3A), whereas while no effect was observed on sedentary rats treated with TT (Fig. 3A). High intensity exercise significantly increased the protein levels of gastrocnemius IGF-1R in the $\mathrm{E}$ and $\mathrm{E}+\mathrm{TT}$ groups compared with the control group $(\mathrm{P}<0.05$ and $\mathrm{P}<0.01$, respectively; Fig. 3B) and treatment with TT extracts resulted in a greater increase than exercise alone ( $\mathrm{P}<0.05$; Fig. 3B). Treatment with TT extracts also increased gastrocnemius IGF-1R protein levels in sedentary rats compared with the control $(\mathrm{P}<0.05$; Fig. 3B), which suggested that TT extracts increased gastrocnemius IGF-1R protein expression levels in sedentary rats and in rats undergoing high intensity exercise.

\section{Discussion}

Our previous study demonstrated that TT extracts promote the performance of rats undergoing 5 weeks of high intensity exercise, reflected by the extension in the time to exhaustion from 90 min to $\sim 150$ min (a 1.7-fold increase compared with the E group), which may be associated with the TT-induced increase of plasma testosterone (18). It of note that commercially available TT extracts and TT saponins contained in nutritional supplements recommended for competitive athletes to enhance their performance may be intentionally contaminated with AAS (36), which may result in inadvertent doping in competitive sports. In contrast, TT saponins without any contamination do not lead to positive anti-doping tests (17). To exclude the possibility of AAS contamination, the chemical components of the TT extracts were analyzed by UHPLC-Q-TOF/MS, a popular method to identify medicine components and metabolites (35). Tigogenin and diosgenin, both saponins, were demonstrated account for $\sim 71.35 \%$ of the total peak area, consistent with the promise of the manufacturers: $>70 \%$ TT saponins. In addition, AAS and other associated hormone precursors were not detected in the extracts. Together with the results of our previous study (18), the present findings suggested that TT extracts, in particular TT saponins, are responsible for the physiological and biological effects which lead to the promotion of endurance performance of rats undergoing high intensity exercise. 
A

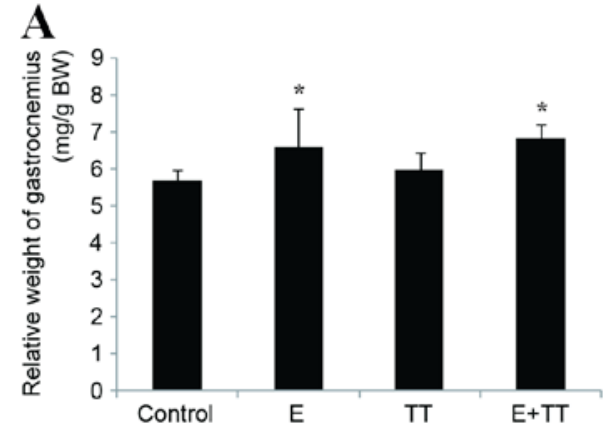

C

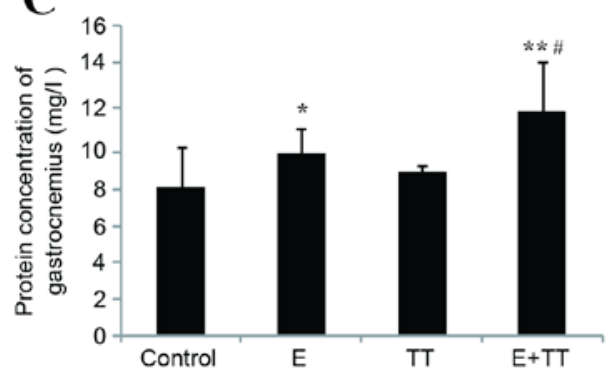

E
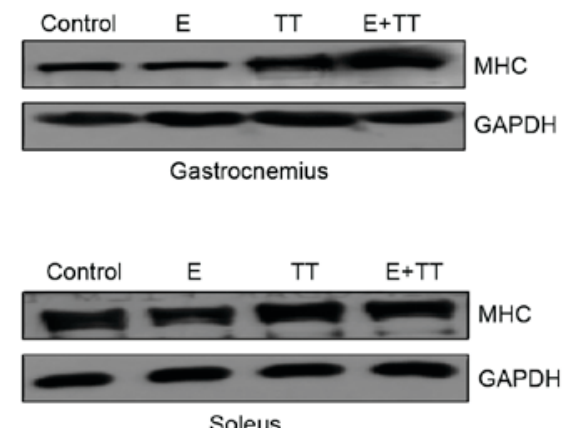

B

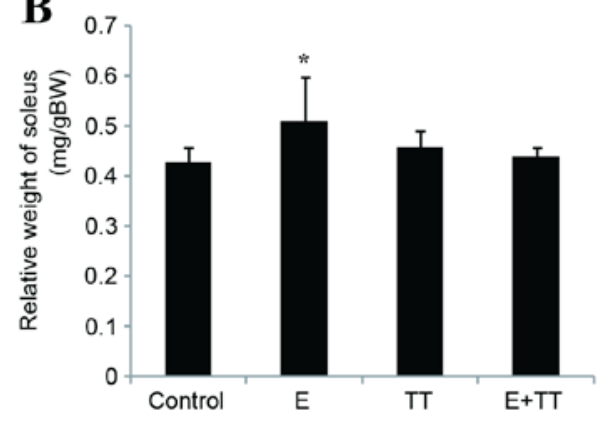

D
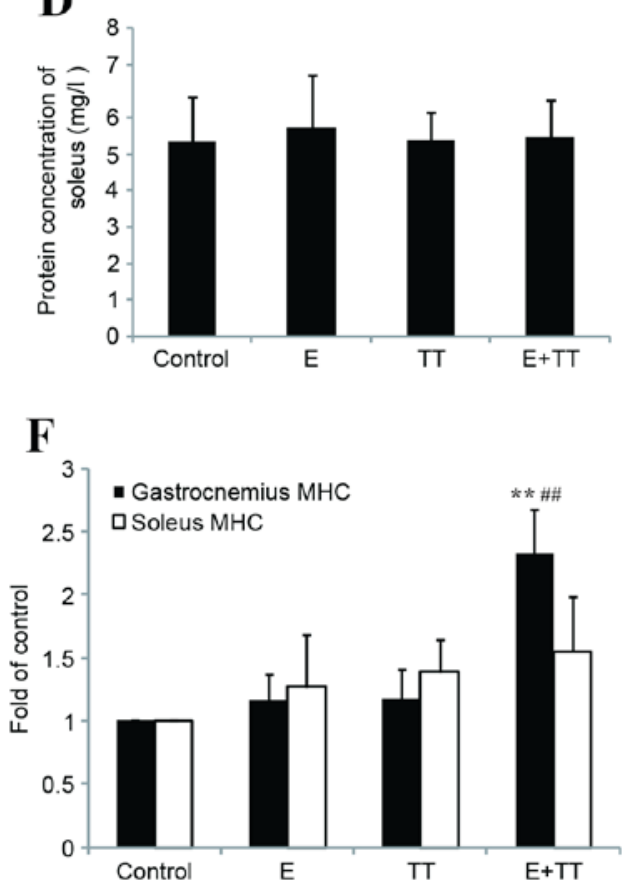

Figure 1. TT extracts increased the relative weight, protein concentration and MHC protein levels of the gastrocnemius, but not the soleus in rats undergoing high intensity exercise. Relative weights of (A) gastrocnemius and (B) soleus following high intensity exercise and treatment with TT extracts. Protein contents of (C) gastrocnemius and (D) soleus following high intensity exercise and treatment with TT extracts. (E) Protein levels of MHC in the gastrocnemius and soleus, determined by western blot, with GAPDH was used as loading control. (F) Relative levels of MHC were quantified, normalized and the values are expressed as the fold of the control. The MHC/GAPDH ratio of the control was identified as 1. Data are expressed as the mean \pm standard deviation of at least three independent experiments. "P $<0.05$ and ${ }^{* * *} \mathrm{P}<0.01$ vs. control; ${ }^{\#} \mathrm{P}<0.05$ and ${ }^{\# \#} \mathrm{P}<0.01$ vs. E group. TT, Tribulus terrestris extracts; MHC, myosin heavy chain; $\mathrm{E}$, high intensity exercise; E+TT, high intensity exercise plus TT.

A

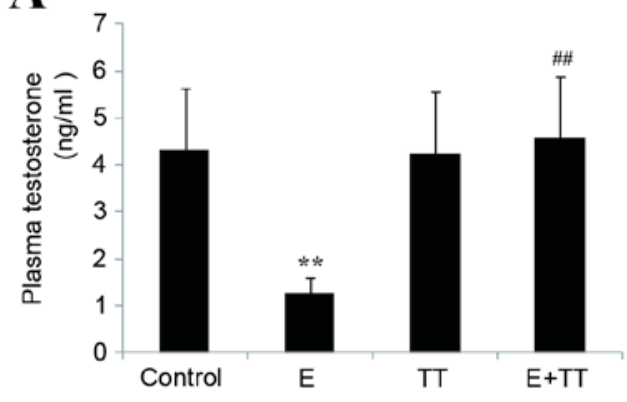

B

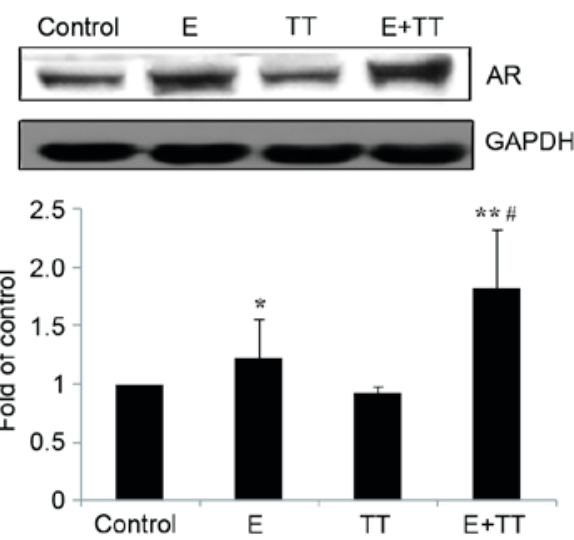

Figure 2. TT extracts increased plasma testosterone levels and AR protein levels in the gastrocnemius of rats undergoing high intensity exercise. (A) Plasma testosterone levels were detected by ELISA. (B) AR protein levels in the gastrocnemius were determined by western blotting and GAPDH was used as loading control. The AR/GAPDH ratio of the control was identified as 1. The relative levels of AR were quantified, normalized and the values are expressed as the fold of the control and the mean \pm standard deviation of at least three independent experiments. ${ }^{*} \mathrm{P}<0.05$ and ${ }^{* * *} \mathrm{P}<0.01$ vs. control; ${ }^{\#} \mathrm{P}<0.05$ and ${ }^{\# \#} \mathrm{P}<0.01$ vs. E group. TT, Tribulus terrestris extracts; AR, androgen receptor; E, high intensity exercise; E+TT, high intensity exercise plus TT. 

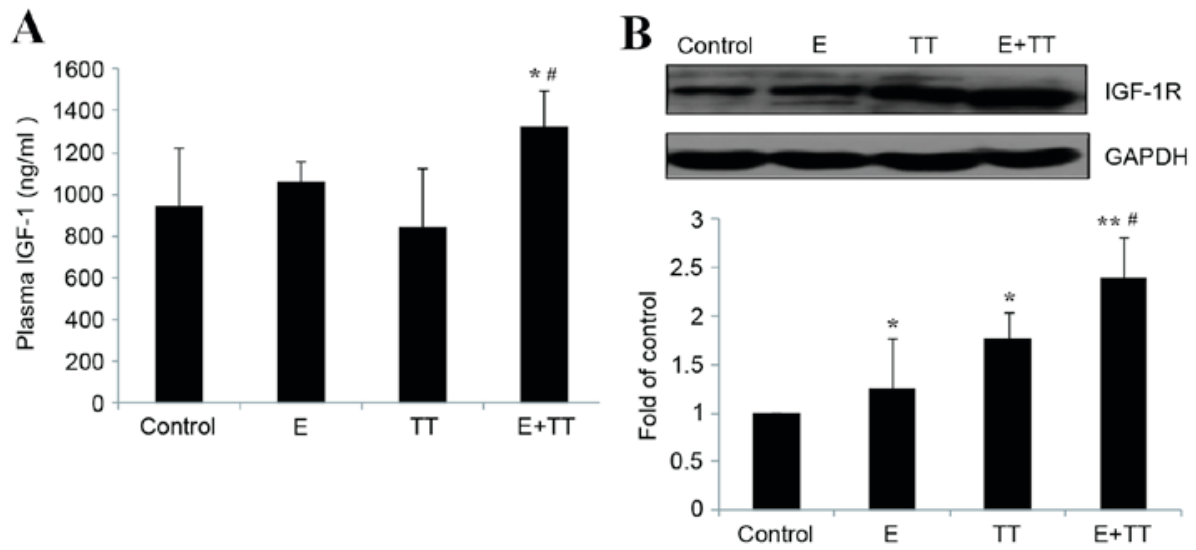

Figure 3. TT extracts increased plasma IGF-1 levels and gastrocnemius IGF-1R protein levels in rats undergoing high intensity exercise. (A) IGF-1 plasma levels were detected by ELISA. (B) Gastrocnemius IGF-1R protein levels were determined by western blotting and GAPDH was used as loading control. Similar results were obtained from three independent experiments. The IGF-1R/GAPDH ratio of the control was identified as 1 . The relative levels of IGF-1R were quantified, normalized and the values are expressed as the fold of the control. ${ }^{*} \mathrm{P}<0.05$ and ${ }^{* *} \mathrm{P}<0.01$ vs. control; ${ }^{*} \mathrm{P}<0.05$ vs. E group. TT, Tribulus terrestris extracts; IGF-1, insulin growth factor-1; IGF-1R, IGF-1 receptor; E, high intensity exercise; E+TT, high intensity exercise plus TT.

In the present study, the beneficial effects of TT extracts on rats undergoing high intensity exercise were demonstrated by improving weight gain and increasing protein content, and the MHC protein levels in the gastrocnemius. It has been demonstrated that exercise promoted contractive muscle hypertrophy, reflected by the increases of the protein synthesis and the weight of these muscles, which may contribute to enhanced exercise performance. MHC constitutes part of the myosin contractile protein in skeletal muscle. The content of MHC protein is used to reflect myosin quantity, as MHC is the major determinant in the speed of contraction of skeletal muscle, contributing to contractile forces and performance $(37,38)$. The intensity of the exercise protocol used in the present study was increased gradually from moderate (week 1-2) to high (week 3-5), resulting in increased protein synthesis and increased relative weight, but not increased MHC protein levels of the gastrocnemius (fast twitch fiber), while only increasing of the relative weight of the soleus (slow twitch fiber). Treatment with TT extracts resulted in further increases of protein content and MHC protein levels in the gastrocnemius while no effect was demonstrated in the soleus, which may explain the increased effect of TT extracts on the body weight and performance of rats undergoing high intensity exercise.

Testosterone exerts its biological function by binding to $\mathrm{AR}$, its cognate receptor. A previous report emphasized the importance of increased AR levels in exercise-induced muscle hypertrophy (26). Mitchell et al (39) demonstrated a correlation between AR protein content and fiber hypertrophy despite no statistical significance in adult males undertaking resistance training. Endurance exercise increased the AR binding capacity of slow twitch fibers and resistance exercise upregulated the AR binding capacity of fast twitch fibers (40). The findings of the present study demonstrated that high intensity exercise led to increased AR expression in the gastrocnemius (fast twitch fiber), which was further increased following treatment with TT extracts. These findings combined with the data that TT extracts induced an increase in testosterone levels, support the hypothesis that the AR signaling pathway is involved in the improved performance of rats undergoing high intensity exercise with administration of TT extracts. The results of the present study are of particular physiological significance as to the best of our knowledge, this is the first report to demonstrate that TT extracts not only increase testosterone levels but also result in an increase of AR in skeletal muscles. In addition, TT extracts do not affect plasma testosterone levels or gastrocnemius AR levels in sedentary rats. The reason that TT saponins have no effect on testosterone and AR in non-trained rats but have significant effect on rats undergoing high intensity training remains to be elucidated.

IGF-1 is associated with muscle mass, conservation of the musculoskeletal system, metabolic rate and muscle strength $(41,42)$. The functions of IGF-1 in the blood and tissues are mediated through binding to tissue IGF-1R (43). IGF-1 is involved in mediating the effects of testosterone on skeletal muscle progenitor cell growth and differentiation in vitro through mediation of IGF-1R (30). However, in MKR mice, which express a dominant negative form of the IGF-1R in their skeletal muscle fibers, IGF-1R signaling does not appear to be obligatory for mediating the anabolic effects of testosterone (30). The results of the present study demonstrated that, accompanied by a significant increase of testosterone, a significant increase in plasma IGF-1 levels and a further increase of the expression of IGF-1R in the gastrocnemius was induced by TT extracts in rats undergoing high intensity exercise. This indicates that IGF-1/IGF-1R may be the primary target of testosterone and one of the major reasons for the beneficial effects of TT extracts. To the best of our knowledge, this is the first report to demonstrate that TT extracts activated the IGF-1/IGF-1R signaling pathway in skeletal muscles.

The present study remains a descriptive study and the present conclusion will be fully supported if it is demonstrated that administration of TT extracts does not increase the performance of rats undergoing high intensity exercise following blocking increases of AR and IGF-1R in the gastrocnemius.

The findings of the present study indicated that the effect of TT extracts on the performance of rats undergoing high 
intensity exercise may be attributed to the induced increases of circulating testosterone and IGF-1, as well as the increases of AR and IGF-1R protein expression levels in the gastrocnemius, resulting in increased muscle weight and myosin content in the gastrocnemius.

\section{Acknowledgements}

The present study was supported by the National Natural Science Foundation of China (grant no. 31271274) and the Key Laboratory of Exercise and Health Sciences, Shanghai University of Sport, Ministry of Education.

\section{References}

1. Velders M and Diel P: How sex hormones promote skeletal muscle regeneration. Sports Med 43: 1089-1100, 2013.

2. Schoenfeld BJ: Postexercise hypertrophic adaptations: A reexamination of the hormone hypothesis and its applicability to resistance training program design. J Strength Cond Res 27: 1720-1730, 2013.

3. Wood RI and Stanton SJ: Testosterone and sport: Current perspectives. Horm Behav 61: 147-155, 2012.

4. Di Luigi L, Romanelli F, Sgrò P and Lenzi A: Andrological aspects of physical exercise and sport medicine. Endocrine 42 278-284, 2012.

5. Bassil N, Alkaade S and Morley JE: The benefits and risks of testosterone replacement therapy: A review. Ther Clin Risk Manag 5: 427-448, 2009.

6. El-Tantawy WH, Temraz A and El-Gindi OD: Free serum testosterone level in male rats treated with Tribulus alatus extracts. Int Braz J Urol 33: 554-559, 2007.

7. Gauthaman K and Ganesan AP: The hormonal effects of Tribulus terrestris and its role in the management of male erectile dysfunction-anevaluation using primates, rabbit and rat. Phytomedicine 15: 44-54, 2008.

8. Qureshi A, Naughton DP and Petroczi A: A systematic review on the herbal extract Tribulus terrestris and the roots of its putative aphrodisiac and performance enhancing effect. J Diet Suppl 11: 64-79, 2014.

9. Singh S, Nair V and Gupta YK: Evaluation of the aphrodisiac activity of Tribulus terrestris Linn. In sexually sluggish male albino rats. J Pharmacol Pharmacother 3: 43-47, 2012.

10. Chhatre S, Nesari T, Somani G, Kanchan D and Sathaye S: Phytopharmacological overview of Tribulus terrestris. Pharmacogn Rev 8: 45-51, 2014.

11. Brown GA, Vukovich MD, Martini ER, Kohut ML, Franke WD, Jackson DA and King DS: Effects of androstenedione-herbal supplementation on serum sex hormone concentrations in 30- to 59-year-old men. Int J Vitam Nutr Res 71: 293-301, 2001.

12. Gauthaman K, Adaikan PG and Prasad RN: Aphrodisiac properties of Tribulus Terrestris extract (Protodioscin) in normal and castrated rats. Life Sci 71: 1385-1396, 2002.

13. Martino-Andrade AJ, Morais RN, Spercoski KM, Rossi SC, Vechi MF, Golin M, Lombardi NF, Greca CS and Dalsenter PR: Effects of Tribulus terrestris on endocrine sensitive organs in male and female Wistar rats. J Ethnopharmacol 127: 165-170, 2010.

14. Rogerson S, Riches CJ, Jennings C, Weatherby RP, Meir RA and Marshall-Gradisnik SM: The effect of five weeks of Tribulus terrestris supplementation on muscle strength and body composition during preseason training in elite rugby league players. J Strength Cond Res 21: 348-353, 2007.

15. Antonio J, Uelmen J, Rodriguez R and Earnest C: The effects of Tribulus terrestris on body composition and exercise performance in resistance-trained males. Int $\mathbf{J}$ Sport Nutr Exerc Metab 10: 208-215, 2000

16. Brown GA, Vukovich MD, Reifenrath TA, Uhl NL, Parsons KA, Sharp RL and King DS: Effects of anabolic precursors on serum testosterone concentrations and adaptations to resistance training in young men. Int J Sport Nutr Exerc Metab 10 340-359, 2000.

17. Saudan C, Baume N, Emery C, Strahm E and Saugy M: Short term impact of Tribulus terrestris intake on doping control analysis of endogenous steroids. Forensic Sci Int 178: e7-e10, 2008.
18. Yin L, Wang X, Cao X and Wang X: The effects of tribulus terrestris on the time of exhaustion in rats with high intensity training and its mechanism. J Shanghai University Sport 37: 73-77, 2013

19. Matsumoto T, Sakari M, Okada M, Yokoyama A, Takahashi S, Kouzmenko A and Kato S: The androgen receptor in health and disease. Annu Rev Physiol 75: 201-224, 2013.

20. Vingren JL, Kraemer WJ, Hatfield DL, Volek JS, Ratamess NA, Anderson JM, Häkkinen K, Ahtiainen J, Fragala MS, Thomas GA, et al: Effect of resistance exercise on muscle steroid receptor protein content in strength-trained men and women. Steroids 74: 1033-1039, 2009.

21. Safarinejad MR, Azma K and Kolahi AA: The effects of intensive, long-term treadmill running on reproductive hormones, hypothalamus-pituitary-testis axis, and semen quality: A randomized controlled study. J Endocrinol 200: 259-271, 2009.

22. Lee WJ, Thompson RW, McClung JM and Carson JA: Regulation of androgen receptor expression at the onset of functional overload in rat plantaris muscle. Am J Physiol Regul Integr Comp Physiol 285: R1076-R1085, 2003.

23. Aizawa K, Iemitsu M, Maeda S, Otsuki T, Sato K, Ushida T, Mesaki $\mathrm{N}$ and Akimoto T: Acute exercise activates local bioactive androgen metabolism in skeletal muscle. Steroids 75: 219-223, 2010.

24. Hulmi JJ, Ahtiainen JP, Selänne H, Volek JS, Häkkinen K, Kovanen V and Mero AA: Androgen receptors and testosterone in men-effects of protein ingestion, resistance exercise and fiber type. J Steroid Biochem Mol Biol 110: 130-137, 2008.

25. Ratamess NA, Kraemer WJ, Volek JS, Maresh CM, Vanheest JL, Sharman MJ, Rubin MR, French DN, Vescovi JD, Silvestre R, et al: Androgen receptor content following heavy resistance exercise in men. J Steroid Biochem Mol Biol 93: 35-42, 2005.

26. Ahtiainen JP, Hulmi JJ, Kraemer WJ, Lehti M, Nyman K, Selänne H, Alen M, Pakarinen A, Komulainen J, Kovanen V, et al: Heavy resistance exercise training and skeletal muscle androgen receptor expression in younger and older men. Steroids 76: 183-192, 2011.

27. McMahon CD, Chai R, Radley-Crabb HG, Watson $T$, Matthews KG, Sheard PW, Soffe Z, Grounds MD and Shavlakadze T: Lifelong exercise and locally produced insulin-like growth factor-1 (IGF-1) have a modest influence on reducing age-related muscle wasting in mice. Scand J Med Sci Sports 24: e423-e435, 2014

28. Luo L, Lu AM, Wang Y, Hong A, Chen Y, Hu J, Li X and Qin ZH: Chronic resistance training activates autophagy and reduces apoptosis of muscle cells by modulating IGF-1 and its receptors, Akt/mTOR and Akt/FOXO3a signaling in aged rats. Exp Gerontol 48: 427-436, 2013.

29. Kim J, Wende AR, Sena S, Theobald HA, Soto J, Sloan C, Wayment BE, Litwin SE, Holzenberger M, LeRoith D and Abel ED: Insulin-like growth factor I receptor signaling is required for exercise-induced cardiac hypertrophy. Mol Endocrinol 22: 2531-2543, 2008.

30. Serra C, Bhasin S, Tangherlini F, Barton ER, Ganno M, Zhang A, Shansky J, Vandenburgh HH, Travison TG, Jasuja R and Morris C: The role of GH and IGF-I in mediating anabolic effects of testosterone on androgen-responsive muscle. Endocrinology 152: 193-206, 2011.

31. Knapczyk-Stwora K, Grzesiak M, Duda M, Koziorowski M and Slomczynska M: Effect of flutamide on folliculogenesis in the fetal porcine ovary-regulation by Kit ligand/c-Kit and IGF1/IGF1R systems. Anim Reprod Sci 142: 160-167, 2013.

32. Lee SH, Johnson D, Luong R and Sun Z: Crosstalking between androgen and PI3K/AKT signaling pathways in prostate cancer cells. J Biol Chem 290: 2759-2768, 2015.

33. Kretzschmar K, Cottle DL, Schweiger PJ and Watt FM: The androgen receptor antagonizes Wnt/ $\beta$-Catenin signaling in epidermal stem cells. J Invest Dermatol 135: 2753-2763, 2015.

34. Tarulli GA, Butler LM, Tilley WD and Hickey TE: Bringing androgens up a NOTCH in breast cancer. Endocr Relat Cancer 21: T183-T202, 2014.

35. Wu JL, Leung EL, Zhou H, Liu L and Li N: Metabolite analysis of toosendanin by an ultra-high performance liquid chromatography-quadrupole-time of flight mass spectrometry technique. Molecules 18: 12144-12153, 2013.

36. Cavalcanti Gde A, Leal FD, Garrido BC, Padilha MC and de Aquino Neto FR: Detection of designer steroid methylstenbolone in 'nutritional supplement' using gas chromatography and tandem mass spectrometry: Elucidation of its urinary metabolites. Steroids 78: 228-233, 2013. 
37. Kim JH and Thompson LV: Non-weight bearing-induced muscle weakness: The role of myosin quantity and quality in MHC type II fibers. Am J Physiol Cell Physiol 307: C190-C194, 2014.

38. Schilling BK, Fry AC, Chiu LZ and Weiss LW: Myosin heavy chain isoform expression and in vivo isometric performance: A regression model. J Strength Cond Res 19: 270-275, 2005.

39. Mitchell CJ, Churchward-Venne TA, Bellamy L, Parise G, Baker SK and Phillips SM: Muscular and systemic correlates of resistance training-induced muscle hypertrophy. PLoS One 8: e78636, 2013.

40. Deschenes MR, Maresh CM, Armstrong LE, Covault J, Kraemer WJ and Crivello JF: Endurance and resistance exercise induce muscle fiber type specific responses in androgen binding capacity. J Steroid Biochem Mol Biol 50: 175-179, 1994.
41. Frystyk J: Exercise and the growth hormone-insulin-like growth factor axis. Med Sci Sports Exerc 42: 58-66, 2010.

42. Gibney J, Healy ML and Sönksen PH: The growth hormone/insulin-like growth factor-I axis in exercise and sport. Endocr Rev 28: 603-624, 2007.

43. Philippou A, Halapas A, Maridaki M and Koutsilieris $M$ Type I insulin-like growth factor receptor signaling in skeletal muscle regeneration and hypertrophy. J Musculoskelet Neuronal Interact 7: 208-218, 2007. 